\title{
Adrenocortical Carcinoma with a Giant Pericardial Mass
}

\author{
Akio NaKata, Shinji Yagi, Koichi Oyama, Hiroshi Kida and Goro SugioKa
}

\begin{abstract}
A patient with a history of adrenocortical carcinoma presented with massive pericardial effusion and a giant pericardial mass. Death resulted from cardiac tamponade. At autopsy a large necrotic mass was histologically similar to the original adrenocortical carcinoma. We present this case and review the literature on metastatic pericardial disease.

(Internal Medicine: 32: 438-440, 1993)
\end{abstract}

Key words: cardiac tamponade, pericardial metastasis, CT scan, Mitotane

\section{Introduction}

Although pericardial metastases are not unusual, pericardial masses are rarely encountered(1-3). We present a rare case of adrenocortical carcinoma in which cardiac tamponade and a giant mass in the pericardial cavity developed, and review the literature on metastatic epicardial masses.

\section{Case Report}

A 53-year-old woman was admitted to the hospital on May 30,1991 , with increasing dyspnea and progressive cardiomegaly. In 1988, she had undergone left adrenalectomy and nephrectomy for a non-functioning adrenocortical carcinoma with metastasis to the left lung. Postoperatively she received chemotherapy (Mitotane $1,500 \mathrm{mg} /$ day) with no change in size of the lung mass.

On admission, the blood pressure was $122 / 62 \mathrm{mmHg}$ and the heart rate was $108 / \mathrm{min}$, with a paradoxical pulse. There were no rales in the lung fields. Heart sounds were normal without murmurs or a pericardial friction rub. Facial edema was evident but there was no leg edema.

Chest radiographs revealed marked cardiac enlargement and a mass in the left middle lung field. A 12-lead electrocardiogram showed sinus tachycardia, depressed ST segments and inverted T waves in leads I, II, III, aVF and V4-6. Echocardiography disclosed a solid mass and a large effusion in the pericardial cavity. Computed tomography (CT scan) of the chest disclosed an enhancing mass in the pericardial cavity (Fig. 1A). Metastasis of adrenocortical carcinoma to the epicardium was suspected. The right atrial mean pressure was $18 \mathrm{mmHg}$, and the mean pulmonary capillary wedge pressure was $21 \mathrm{mmHg}$. The cardiac index was $1.64 \mathrm{~L} / \mathrm{min} / \mathrm{m}^{2}$.

Laboratory data showed a leukocyte count of 10,400 , C- reactive protein of $9.1 \mathrm{mg} / \mathrm{dl}$, and an erythrocyte sedimentation rate of $60 \mathrm{~mm} / \mathrm{hr}$. In addition, a slight elevation in transaminase probably due to passive liver congestion was found. Neuronspecific enolase was as high as $66.2 \mathrm{ng} / \mathrm{ml}$. The urinary 17 hydroxycorticosteroid and 17-ketosteroid were normal. The pericardial fluid was bloody with a protein concentration of $5 \mathrm{~g} /$ $\mathrm{dl}$ and was characterized as an exudate.

Surgical drainage of the pericardial cavity was performed on June 2 and a total of 2,000 cc was evacuated. Doxycycline and minocycline were then infused into the pericardial cavity. When drainage stopped, the pericardial tube was removed. No increase in pericardial effusion was subsequently observed.

The pericardial mass increased its size within 3 months, with the CT scan demonstrating an area of low density in the mass, which was considered to reflect necrosis (Fig. 1B). The paradoxical pulse recurred on July 29 and the low cardiac output decreased further. The patient died on August 25.

Autopsy revealed no local recurrence of adrenocortical carcinoma, but multiple metastatic foci were found in the lungs. The heart weighed 1,300 gm. The space between the epicardium and the myocardium was infiltrated with necrotic tumor (Fig. 2A). The histologic picture of the mass was consistent with the previous adrenocortical carcinoma, with typical large clear cells and multiple mitotic figures (Fig. 2B). The pericardial mass was, therefore, diagnosed as a metastatic adrenal cortical carcinoma.

\section{Discussion}

Cardiac metastasis of a malignant tumor is not rare, with a reported incidence of 9.7-12.2\% (4). Malignant tumors which commonly metastasize to the heart include lung cancer, breast cancer, malignant lymphoma, leukemia and hypernephroma. The pericardium is most common site of cardiac metastasis

From the Department of Cardiology, Kanazawa National Hospital, Kanazawa Received for publication June 2, 1992; Accepted for publication April 19, 1993

Reprint requests should be addressed to Dr. Akio Nakata, the First Department of Internal Medicine, Kanazawa University, 13-1 Takaramachi, Kanazawa 920, Japan 


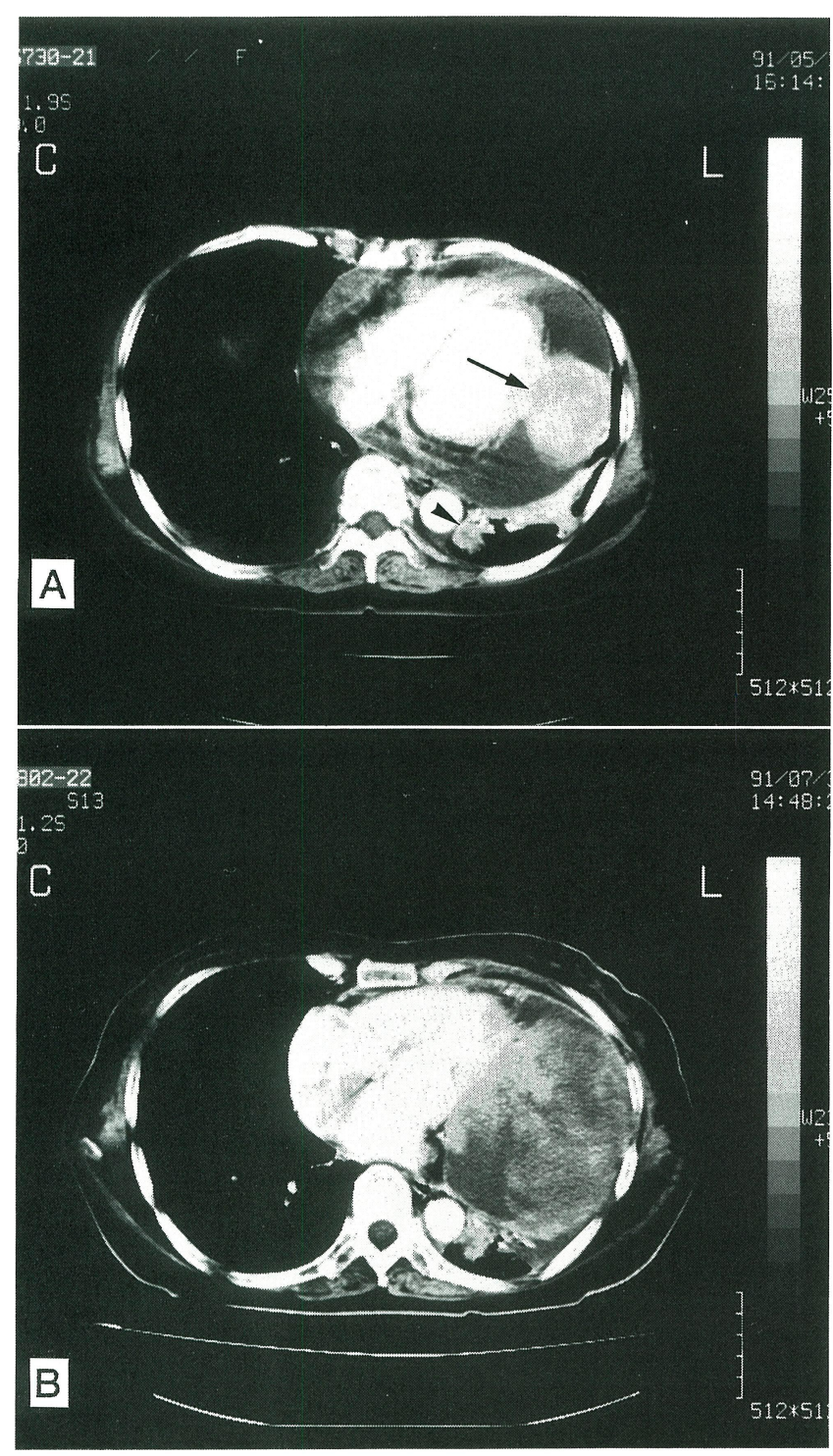

Fig. 1. Computerized tomographic scan with contrast medium on admission demonstrating massive pericardial effusion and a solid mass (arrow) in the pericardium. A nodule (arrowhead) is also noted in the left lung (A). Three months later, the pericardial mass had become enlarged, resulting in an area of low density in the pericardium (B).

followed in frequency by the myocardium. Pericardial effusion is frequently an accompanying occurrence of pericardial metastasis (5). If it is advanced, cardiac tamponade ensues, requiring drainage or pericardiocentesis. In rare cases, epicardial metastases produce a pathologic state similar to constrictive pericarditis (4). However there have been no prior reports of such a giant pericardial mass demonstrated radiologically $(1-3$, $6,7)$.

Adrenocortical carcinoma is a relatively rare malignant tumor, with a frequency of $0.0002 \%(1,8)$. Furthermore, nonfunctioning adrenocortical carcinoma is said to be less frequent than the secretary type. This may account for the paucity of
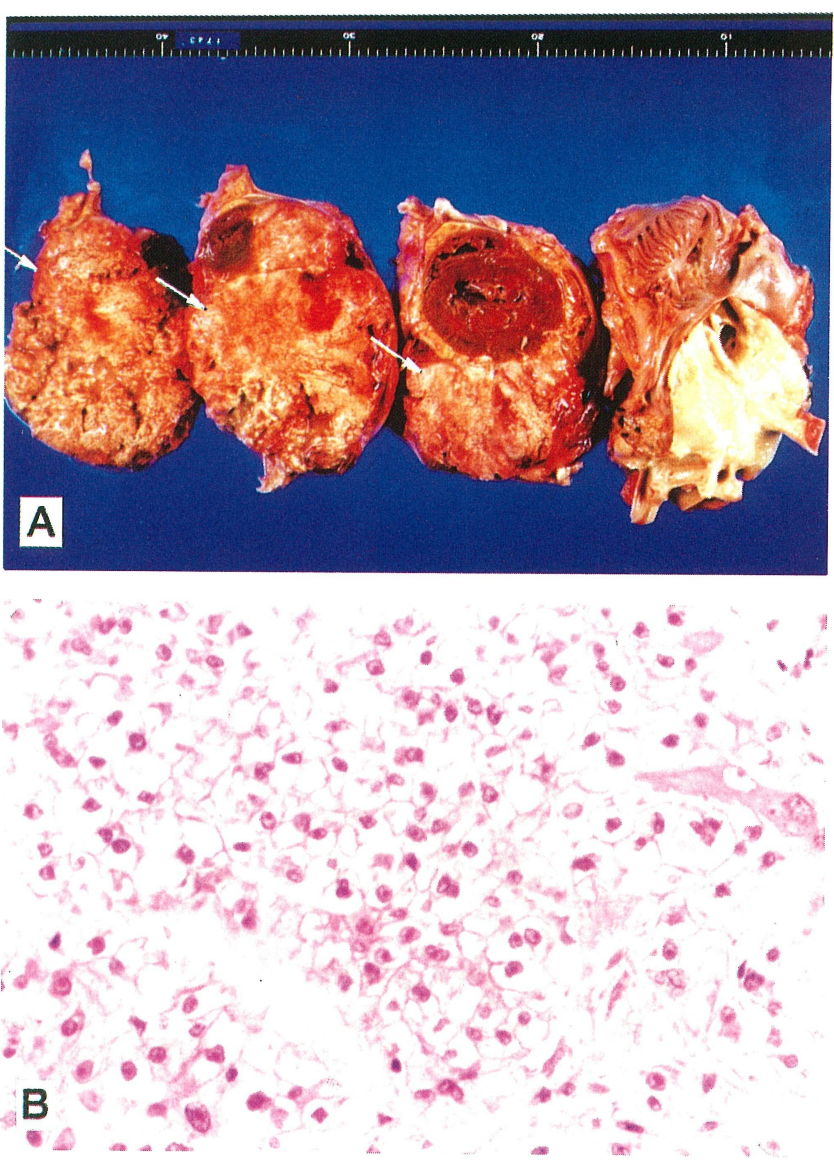

Fig. 2. Section of the heart demonstrating a giant mass (arrow) in the pericardium with a necrotic component (A). Histologic picture reveals clear cells with multiple mitotic figures resembling the adrenocortical carcinoma removed previously $(\mathrm{B})(\times 400)$.

reports of cardiac metastasis of adrenocortical carcinoma. Adrenocortical carcinoma is a highly malignant tumor (2). From $30 \%$ to $50 \%$ of patients present with metastases at diagnosis, and in only $4-7 \%$ is the tumor confined to the adrenal gland $(3,9)$.

In the present case, based on the existence of mediastinal lymph node metastasis, a lymphatic route was suspected. Although the mechanism of formation of a giant tumor mass in the pericardial cavity is unclear, some relationship to such high malignancy is suspected.

\section{References}

1) Hajjar RA, Hickey RC, Samaan NA. Adrenal cortical carcinoma. Cancer 35: 549, 1975.

2) King DR, Lack EE. Adrenal cortical carcinoma. A clinical and pathologic study of 49 cases. Cancer 44: 239, 1979.

3) Didolkar MS, Bescher RA, Elias EG, Moore RH. Natural history of adrenal cortical carcinoma: A clinicopathologic study of 42 patients. Cancer 47: 2153, 1981.

4) Hanfling SM. Metastatic cancer to the heart: Review of the literature and report of 127 cases. Circulation 22: 474, 1960.

5) Deloran LT, Jesse EE, Richard WPA. Secondary malignant tumors of the 


\section{NaKata et al}

pericardium. Circulation 26: 228, 1962.

6) Brian WP, Sinda BD, Christopher W, Claude RJ. Right ventricular inflow and outflow obstruction due to adrenal cell carcinoma. Catheterization and Cardiovascular Diagnosis 12: 51, 1986.

7) Paul S, Eberhard D, Anupam B, Walter H, Helmut U. Adrenal cortical carcinoma: Preoperative demonstration of right atrial extension by sonography and computed tomography. J Urol 133: 260, 1985.

8) Hutter AM, Kayhoe DE. Adrenal cortical carcinoma. Am J Med 41: 572, 1966.

9) Sullivan M, Bolieau M, Hodges CV. Adrenal cortical carcinoma. J Urol 120: 660,1978 